\title{
ANTERIOR MARGINAL SEPARATION OF THE MENISCUS OF THE KNEE
}

\author{
H. H. Boucher, Vancouver, Canada
}

Not infrequently an apparently normal meniscus is removed. In a series of seventy-seven operations Bristow (1925) reported finding a normal cartilage in thirteen. in five of which there was hypertrophy of the fat pad. In a further seven cases the lesion was obscure, so that findings were unsatisfactory in twenty out of seventy-seven operations. Since the pre-operative diagnosis of internal derangement had seemed almost certain Bristow suggested that nipping of the retropatellar fat pad was the cause of the trouble. In discussing this paper Jones (1925) recalled that in a personal series of 117 operations he had found eight menisci to be loosely bound circumferentially, with no other abnormality. He had no doubt that a "preternaturally" mobile anterior horn existed and could give rise to symptoms and that this condition was not uncommon. Parry, Nichols and Lewis (1958) found a rather high incidence of loose and otherwise normal cartilages in a series of 1,275 meniscectomies. and Smillie (1962) found 4 per cent of normal cartilages in a series of 4,500 meniscectomies.

Helfet (1959) described the "retracted" meniscus, with definite clinical evidence of derangement and obvious damage to the anterior attachments and body of the meniscus. This is the most important feature of the lesion discussed in this paper in which clinical evidence of it is often slight.

In 1946, in an effort to reduce our own errors, the orthopaedic staff at Shaughnessy Hospital decided to hold a weekly conference on internal derangement of the knee. Patients were examined by all the staff and if it was thought advisable the examination was repeated under general anaesthesia. A record of the diagnoses was made and the operative findings were reported at the conference after operation. It was interesting that we were unable to eliminate errors, and those which occurred in a series of almost 200 operations were reported by Boucher (1950). Two kinds of error seemed to be of particular interest. In seven patients there was no evidence of internal derangement, and localised chondromalacia of the weightbearing surface of a femoral condyle was present in seven other patients in whom the meniscus was apparently normal. As a diagnosis of torn meniscus had been made it was removed in each instance, as recommended by Watson-Jones (1940).

ANATOMY

Loose coronary ligaments attach the convex margins of the menisci to the tibia (Grant 1948) (Fig. 1). As the horns of the lateral meniscus are attached close together and its coronary ligaments are slack, it can slide backwards and forwards on the flat surface of the lateral tibial condyle. In contrast, the horns of the medial meniscus are attached wide apart. and its movement on the concave surface of the medial tibial condyle is more restricted. Moreover, whereas the lateral meniscus moves backwards as a whole, the medial meniscus, being anchored in its posterior half, is unable to do so and the anterior half alone moves backward, causing a well marked kink at its junction with the posterior half.

Stretching or rupture of the marginal attachments of the anterior horn of the meniscus may occur without injury to the body of the meniscus. As its normal elasticity has been lost the meniscus is unable to retrace its steps, so that when extension of the knee takes place, it is nipped between the condyles of the femur and the tibia and may be damaged further (Fisher 1924). This abnormal contact may also produce pressure areas of degeneration in the femoral and tibial condyles.

VOL. 46 B, NO. 3, AUGUST 1964 
When the joint is opened there may be no evidence of looseness or separation of the anterior marginal attachments and the body of the meniscus may be intact. If, however, this condition has been kept in mind, the retraction of the soft tissues beneath the deep fascia may have been noticed when the latter was incised.

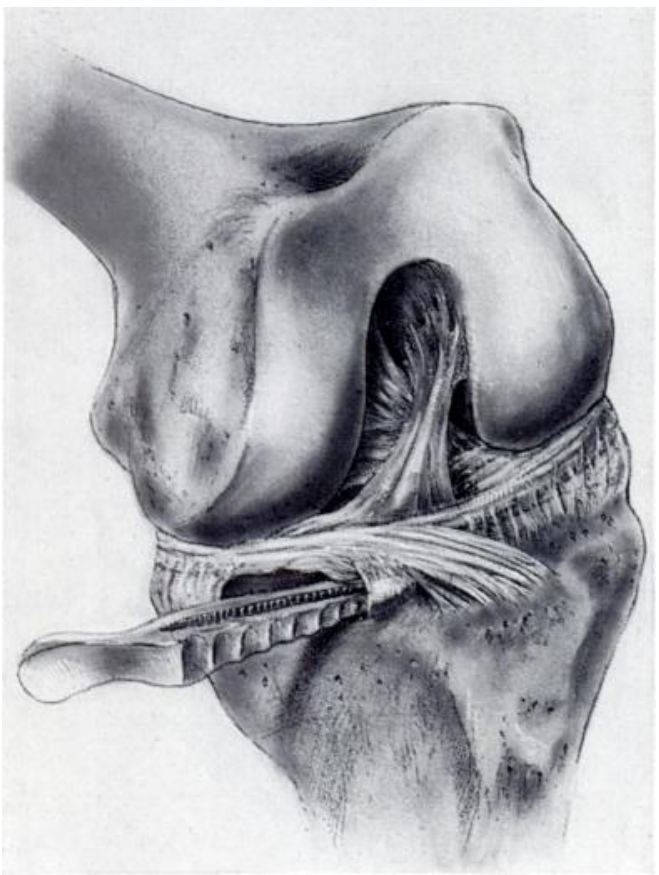

FiG. 1

Showing the loose coronary ligaments. leaving a subfascial space of varying depth containing extra-synovial fat. The displaced meniscus has dragged the synovia and the damaged marginal attachments posteriorly and a forward pull will show the amount of the displacement. The anterior aspect of the tibial condyle can be palpated, covered by a layer of soft tissue, and when this is dissected out, its bare articular surface is seen and the body of the meniscus will be noted lying in close relation to the femoral condyle (Figs. 2 and 3 ). If this derangement has been present for some time, damage to the articular surface of the condyles, mainly the femoral one, may be seen, varying from a faint imprint of the meniscus to the crater-like defect of progressive chondromalacia.

\section{DIAGNOSIS}

Symptoms are those common to internal derangement due to meniscus injury and manipulation may or may not be helpful (McMurray 1937). Circumduction in slight flexion may demonstrate gross anterior relaxation of the coronary ligament. On flexion, the normal anterior anatomical sulcus will be deepened and on extension the meniscus will be displaced forward, bulging under the thumb. If the injury to the coronary ligament is recent, oedema and fibrosis will tend to fill the sulcus and tenderness will be found.

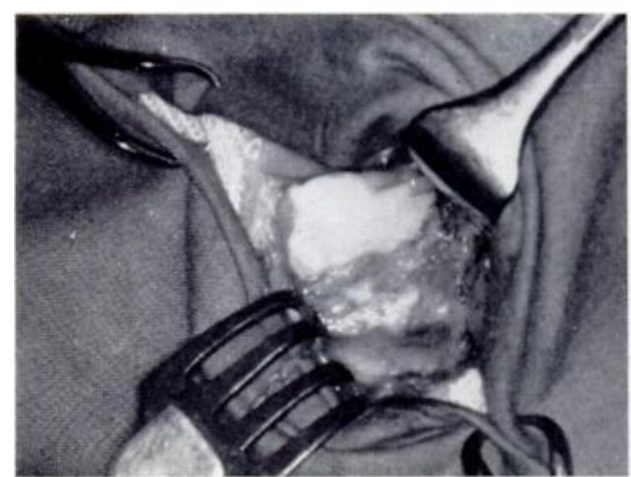

Fig. 2

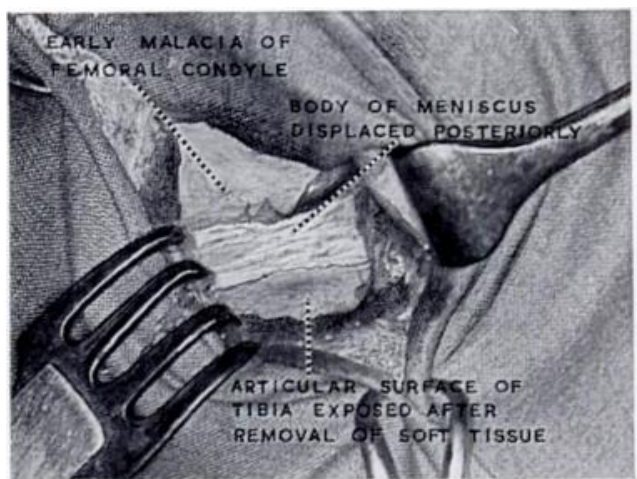

FIG. 3

Figure 2-Retracted soft tissue attached to posteriorly displaced meniscus and covering the articular surface of the tibia. Early malacia of weight-bearing surface of femoral condyle due to pressure contact with meniscus is seen. Figure 3-The lesion further exposed.

In cases of minor strain the diagnosis is difficult and is often not made. The complaints are varied and vague with only slight disability, and the lack of clinical signs may be such as to postpone operation. It is during this period that the area of chondromalacia on the 
weight-bearing surface of the femoral condyle may develop. A further factor contributing to a delay in diagnosis is when an asymptomatic hypermobile meniscus is present in the opposite knee. A major strain may bring the patient to operation, when, in addition to a fresh tear of the meniscus, an area of chondromalacia of long standing may be found with old marginal stretching or separation.

I have studied the findings in a series of approximately 350 operations for internal derangement of the knee. Twenty-seven medial and seven lateral menisci were found to have varying degrees of stretching or tearing of the anterior marginal attachment. In fifteen of these patients an associated tear of the meniscus was found, while in the remainder the body of the meniscus was intact. The posterior displacement of the anterior horn varied from a minor degree to that of major rupture of the marginal attachments. Chondromalacia of the femoral condyle was present in operations on two lateral and ten medial menisci.

\section{SUMMARY}

1. Strain or rupture of the anterior marginal attachments of the meniscus was observed in approximately 10 per cent of a group of patients operated upon for internal derangement. 2. In about half of these a tear of the body of the meniscus was found and it is probable that this tear may have been the cause of the symptoms. In the remainder no injury to the body of the meniscus was found.

3. Recognition may be difficult and delay in diagnosis may be the cause of degenerative joint changes.

4. The condition should be looked for at operation when the meniscus appears to be intact or when the only lesion appears to be an area of chondromalacia on the weight-bearing surface of the femoral condyle.

\section{REFERENCES}

Boucher, H. H. (1950): Errors in Diagnosis of Internal Derangement of the Knee. Paper read at Annual Meeting of the Canadian Orthopaedic Association.

Bristow, W. R. (1925): Internal Derangement of the Knee-Joint. Journal of Bone and Joint Surgery, 7, 413. Fisher, A. G. T. (1924): Internal Derangements of the Knee-Joint. London: H. K. Lewis \& Co. Ltd.

Grant, J. C. B. (1948): A Method of Anatomy. Fourth edition. Baltimore: Williams \& Wilkins Co.

Helfet, A. J. (1959): Mechanism of Derangements of the Medial Semilunar Cartilage and their Management. Journal of Bone and Joint Surgery, 41-B, 319.

JONES, Sir R. (1925): In Discussion of Internal Derangement of the Knee. Journal of Bone and Joint Surgery; 7, 439.

McMurray, T. P. (1937): A Practice of Orthopaedic Surgery. London: Edward Arnold \& Co.

Parry, C. B. Wynn, Nichols, P. J. R., and Lewis, N. R. (1958): Meniscectomy: a Review of 1,723 Cases. Annals of Physical Medicine, 4, 201.

SmiLlie, I. S. (1962): Injuries of the Knee Joint. Third edition. Edinburgh and London: E. \& S. Livingstone Ltd. WATSON-JoneS, Sir R. (1940): Fractures and Other Bone and Joint Injuries. Edinburgh: E. \& S. Livingstone Ltd. 